\title{
Thermal transport signatures of the excitonic transition and associated phonon softening in the layered chalcogenide $\mathrm{Ta}_{2} \mathrm{NiSe}_{5}$
}

\author{
Yuan-Shan Zhang, ${ }^{1}$ Jan A. N. Bruin, ${ }^{1, *}$ Yosuke Matsumoto,,${ }^{1}$ Masahiko Isobe, ${ }^{1}$ and Hidenori Takagi $\circledast^{1,2,3, \dagger}$ \\ ${ }^{1}$ Max Planck Institute for Solid State Research, 70569 Stuttgart, Germany \\ ${ }^{2}$ Institute for Functional Matter and Quantum Technologies, University of Stuttgart, 70569 Stuttgart, Germany \\ ${ }^{3}$ Department of Physics, University of Tokyo, 113-0033 Tokyo, Japan
}

(Received 25 March 2021; revised 4 September 2021; accepted 8 September 2021; published 17 September 2021)

\begin{abstract}
The quasi-one-dimensional layered compound $\mathrm{Ta}_{2} \mathrm{NiSe}_{5}$ has been proposed to undergo a transition to an excitonic insulator at $T_{\mathrm{c}}=326 \mathrm{~K}$. We found a clear anomaly at $T_{\mathrm{c}}$ in the in-plane thermal conductivities both parallel ( $\| a$ ) and perpendicular $(\| c)$ ) to the one-dimensional chains, $\kappa_{\mathrm{a}}$ and $\kappa_{\mathrm{c}}$. While $\kappa_{\mathrm{a}}$ shows a rapid decrease below $T_{\mathrm{c}}, \kappa_{\mathrm{c}}$ shows a pronounced $\mathrm{V}$-shaped suppression centered at $T_{\mathrm{c}}$. We argue that the decrease of $\kappa_{\mathrm{a}}$ represents the suppression of the quasiparticle contribution below $T_{\mathrm{c}}$ due to the excitonic transition. On the other hand, the V-shaped suppression of $\kappa_{\mathrm{c}}$ comes from the enhanced phonon scattering by soft phonons associated with the monoclinic transition with momentum $\mathbf{q} \| c$. The continued suppression of $\kappa_{\mathrm{c}}$ up to an extremely high temperature above $T_{\mathrm{c}}$ suggests the persistence of phonon softening likely coupled to electronic, presumably excitonic, fluctuations.
\end{abstract}

DOI: 10.1103/PhysRevB.104.L121201

The excitonic insulator (EI), in which bound electron-hole pairs form a condensate below a transition temperature $T_{\mathrm{c}}$, had been proposed theoretically more than 50 years ago [1], but its realization in existing materials remains an active subject of investigation. The excitonic transition is anticipated in a nearly zero-gap semiconductor when the exciton binding energy $E_{\mathrm{b}}$ exceeds the one-electron gap $E_{\mathrm{g}}$, or in a semimetal when the attractive interaction between electrons and holes is not screened out well. The realization in bulk materials has been discussed, for example, in $\mathrm{TmSe}_{0.45} \mathrm{Te}_{0.55}$ [2] and $1 T-\mathrm{TiSe}_{2}$ [3], but the evidence for excitonic condensation in these compounds has been far from convincing. The former is a $4 f^{13}$ Mott-like system, not a band semiconductor/semimetal [4], and the latter remains metallic and may suffer from strong screening of electron-hole interactions even below the proposed excitonic transition temperature [5].

Very recently, $\mathrm{Ta}_{2} \mathrm{NiSe}_{5}$ has emerged as a leading candidate for an EI [6-18], which is a direct gap semiconductor/semimetal with $\mathrm{Ta} 5 d$ conduction bands and $\mathrm{Ni} 3 d$ valence bands $[8,15,19]$. It shows a transition to an insulator at $T_{\mathrm{c}}=326 \mathrm{~K}$, which was proposed to be an excitonic transition. The crystal structure of $\mathrm{Ta}_{2} \mathrm{NiSe}_{5}$ is layered, and as shown in Fig. 1(a), each layer consists of an array of Ta and $\mathrm{Ni}$ chains running along the $a$ axis, which renders the system electronically quasi-one-dimensional and gives rise to

\footnotetext{
*j.bruin@fkf.mpg.de

†h.takagi@fkf.mpg.de
}

Published by the American Physical Society under the terms of the Creative Commons Attribution 4.0 International license. Further distribution of this work must maintain attribution to the author(s) and the published article's title, journal citation, and DOI. Open access publication funded by the Max Planck Society. spatially isolated electron and hole chains. The one dimensionality is known to enhance the exciton binding energy [7], which makes $\mathrm{Ta}_{2} \mathrm{NiSe}_{5}$ an ideal platform for the search for an EI. Well below $T_{\mathrm{c}}$, an optical gap of $0.16 \mathrm{eV}$ develops, which is close to the exciton binding energy of $0.25 \mathrm{eV}$ experimentally observed in the optical conductivity spectrum of the sister compound $\mathrm{Ta}_{2} \mathrm{NiS}_{5}$. $\mathrm{Ta}_{2} \mathrm{NiS}_{5}$ has a much larger gap of $0.6 \mathrm{eV}$ and exhibits no clear signatures of an electronic phase transition in electrical transport and optical conductivity $[8,9]$. These observations strongly suggest the excitonic origin of the insulating gap in $\mathrm{Ta}_{2} \mathrm{NiSe}_{5}$ below $T_{\mathrm{c}}$. As a function of the one-electron gap, $E_{\mathrm{g}}$, controlled by pressure and chemical substitution, the transition temperature $T_{\mathrm{c}}$ shows a dome-shaped behavior centered at $E_{\mathrm{g}} \approx 0$, corresponding to pure $\mathrm{Ta}_{2} \mathrm{NiSe}_{5}$ at ambient pressure [8]. This is consistent with what is expected for the canonical EI [1].

The aforementioned experimental results clearly point to the excitonic character of the transition. Nevertheless, it has become increasingly clear that the transition cannot be purely excitonic and that the actual situation is more complicated due to the electron-lattice coupling [19-21], which makes the physics of the putative excitonic transition even more challenging and attractive. The putative excitonic transition at $T_{\mathrm{c}}=326 \mathrm{~K}$ is accompanied by a structural phase transition from an orthorhombic to a monoclinic structure [22,23]. In the monoclinic phase, the $\mathrm{TaSe}_{2}$ chain-ladder unit distorts, such that the Ta chain and the surrounding Se ladders move along the $a$ axis, but in opposite directions [24]. The movement of two neighboring $\mathrm{TaSe}_{2}$ chain-ladder units surrounding the $\mathrm{Ni}$ chain is asymmetric with respect to the $\mathrm{Ni}$ chain. This shear-type distortion with antiferroelectric-type displacement of the Ta and the Se chains does not bring about any superstructure (i.e., $\mathbf{q}=0$ ) but allows for the hybridization of Ta conduction bands and $\mathrm{Ni}$ valence bands around the $\Gamma$ point [19]. The resultant hybridization gap could account for 
(a)

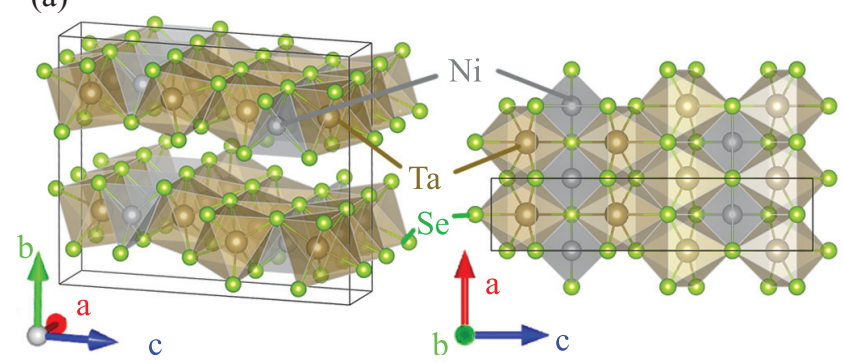

(b)

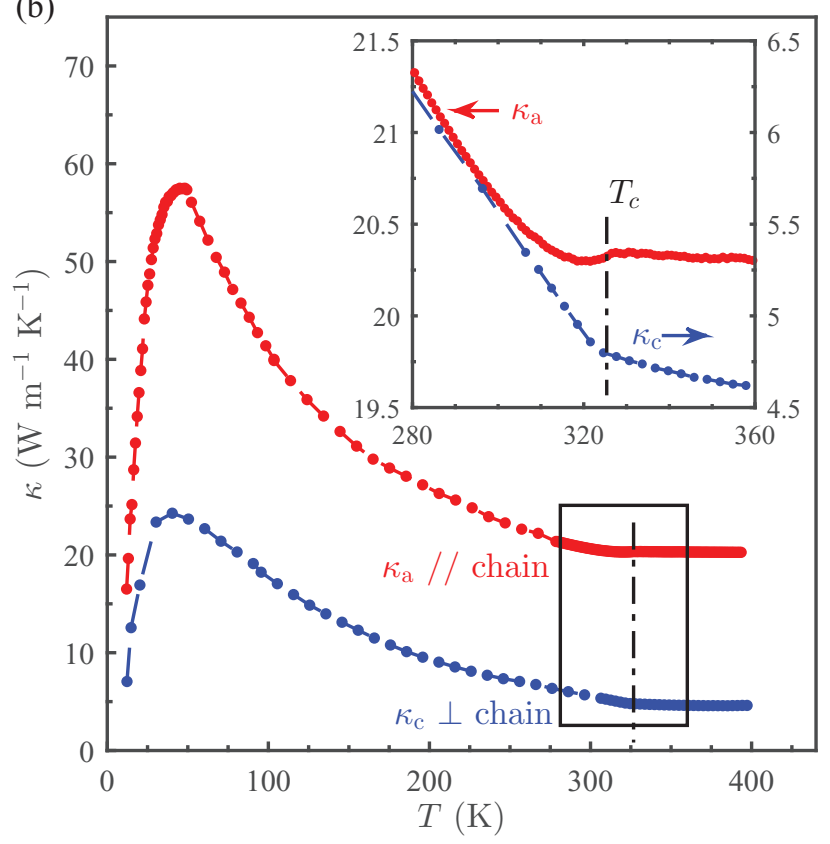

FIG. 1. (a) Crystal structure of $\mathrm{Ta}_{2} \mathrm{NiSe}_{5}$ showing the stack of layers along the $b$ axis (left) and a single layer in the $a$ - $c$ plane consisting of an array of two chains of Ta octahedrally coordinated with $\mathrm{Se}$ and one chain of $\mathrm{Ni}$ in between, tetrahedrally coordinated with $\mathrm{Se}$ (right) [33]. (b) Thermal conductivities $\kappa_{\mathrm{a}}$ with heat current parallel to the chains ( $\| a$; red) and $\kappa_{\mathrm{c}}$ with heat current perpendicular to the chains (\| $c$; blue) as a function of temperature. The dashed black line indicates $T_{\mathrm{c}}=326 \mathrm{~K}$. Inset: Enlarged view of $\kappa_{\mathrm{a}}$ and $\kappa_{\mathrm{c}}$ near $T_{\mathrm{c}}$.

at least a part of the insulating gap and should be one of the important ingredients of the transition at $326 \mathrm{~K}$. A symmetry analysis demonstrated that a finite excitonic order parameter must be accompanied by the monoclinic distortion and hence the admixture of the excitonic gap and the hybridization gap is unavoidable [25].

In this study, we measure the in-plane anisotropic thermal conductivities $\kappa_{\mathrm{a}}(T)$ along the chains $(\| a)$ and $\kappa_{\mathrm{c}}(T)$ perpendicular to the chains $(\| c)$ of $\mathrm{Ta}_{2} \mathrm{NiSe}_{5}$ single crystals in order to unveil the quasiparticle thermal conductivity of the putative EI and the phonon transport around the monoclinic transition. In EIs, quasiparticle transport analogous to Bardeen-CooperSchrieffer superconductors may be expected $[1,26]$. Thermal conductivity is an especially useful probe of the quasiparticle transport in an EI as the cancellation between the electron and the hole quasiparticles does not take place. In addition, thermal conductivity has a large phonon contribution, which is sensitive to the presence of soft phonons through phonon- phonon scatterings $[27,28]$ and therefore may give us hints to decipher the nature of the monoclinic lattice distortion.

We show that $\kappa_{\mathrm{a}}(T)$ along the chains is rapidly suppressed below $T_{\mathrm{c}}$ and that it comes from the decrease in the number of quasiparticles (electrons and holes) below $T_{\mathrm{c}}$, which is expected for a canonical EI [29]. In contrast, $\kappa_{\mathrm{c}}$ perpendicular to the chains shows a V-shaped suppression due to enhanced scattering by soft phonons associated with the orthorhombicto-monoclinic transition with momentum $\mathbf{q}$ perpendicular to the chains $(\| c)$. The strong suppression of $\kappa_{\mathrm{c}}$ persists up to more than $50 \mathrm{~K}$ above $T_{\mathrm{c}}$, which suggests a robust phonon softening likely coupled to excitonic (and hybridization-gap) fluctuations.

Needle-shaped single crystals of $\mathrm{Ta}_{2} \mathrm{NiSe}_{5}$ with a typical size of $4 \mathrm{~mm} \times 1 \mathrm{~mm} \times 0.1 \mathrm{~mm}$ were grown by chemical vapor transport $[8,30]$, with their longest dimension along the $\mathrm{Ta} / \mathrm{Ni}$ chains ( $a$ axis) and their shortest dimension along the interlayer $b$ axis. Rectangular-shaped samples with their long axis along the $a$ and $c$ axes were cut out from the single crystals. The electrical resistivity of the samples was measured by a conventional four-probe technique, while thermal conductivity measurements along the same axes were performed via a "heat-pipe" method [31,32]. The temperature gradients were detected with a differential type-E thermocouple. A transition was observed at $T_{\mathrm{c}}=326 \mathrm{~K}$ as a clear kink in the electrical resistivity along the chain direction $\left(\rho_{\mathrm{a}}\right)$, as reported previously $[8,30]$.

As shown in Fig. 1(b), $\kappa_{\mathrm{a}}(T)$ along the chains $(\| a)$ is a factor of 3-4 larger than $\kappa_{\mathrm{c}}$ perpendicular to the chains $(\| c)$. With increasing temperature from $10 \mathrm{~K}$, both $\kappa_{\mathrm{a}}$ and $\kappa_{\mathrm{c}}$ increase and show a peak around $30-40 \mathrm{~K}$, followed by a gradual decrease that continues above room temperature. This behavior is common for a crystalline solid with phonondominated thermal conductivity $[34,35]$.

At the transition temperature $T_{\mathrm{c}}=326 \mathrm{~K}$, we observe a clear anomaly both in $\kappa_{\mathrm{a}}(T)$ and $\kappa_{\mathrm{c}}(T)$. The anomaly at $T_{\mathrm{c}}$, however, shows up in a contrasted manner between the two directions $a$ and $c$, which can be seen more clearly in the enlarged plot around $T_{\mathrm{c}}$ in the inset of Fig. 1(b). For $\kappa_{\mathrm{a}}(T)$ parallel to the chains, a shoulder structure with a well-defined kink at $T_{\mathrm{c}}$ is observed, followed by an abrupt drop below $T_{\mathrm{c}}$. On further lowering of the temperature, $\kappa_{\mathrm{a}}(T)$ recovers and begins increasing below $\sim 315 \mathrm{~K}$. In contrast, $\kappa_{\mathrm{c}}(T)$ shows a discontinuous change of slope at $T_{\mathrm{c}}$ from a weak increase to a very rapid increase with decreasing temperature, which can be viewed as a V-shaped suppression from the $1 / T$-like increase expected for phonon-dominant, high-temperature thermal transport [34]. The contrast is even clearer in the temperature derivatives, $\partial \kappa_{\mathrm{a}} / \partial T$ and $\partial \kappa_{\mathrm{c}} / \partial T$ [30]. We argue that the contrasted behaviors of the anomaly at $T_{\mathrm{c}}$ between $\kappa_{\mathrm{a}}(T)$ and $\kappa_{\mathrm{c}}(T)$ come from the predominant electronic and phonon contributions in the temperature dependence of $\kappa_{\mathrm{a}}(T)$ and $\kappa_{\mathrm{c}}(T)$, respectively, near $T_{\mathrm{c}}$, which allows us to discuss the behaviors of quasiparticles (electrons and holes) and phonons around $T_{\mathrm{c}}$ separately.

The measured thermal conductivity $\kappa$ comprises contributions from electrons, $\kappa^{(\mathrm{e})}$, and from phonons, $\kappa^{(\mathrm{ph})}$. Around $T_{\mathrm{c}}, \kappa^{(\mathrm{e})}$ can be estimated from the electrical conductivity $\sigma$ using the Wiedemann-Franz (W-F) law. The W-F law is a realistic approximation around $T_{\mathrm{c}}$, as large-angle electron-phonon 


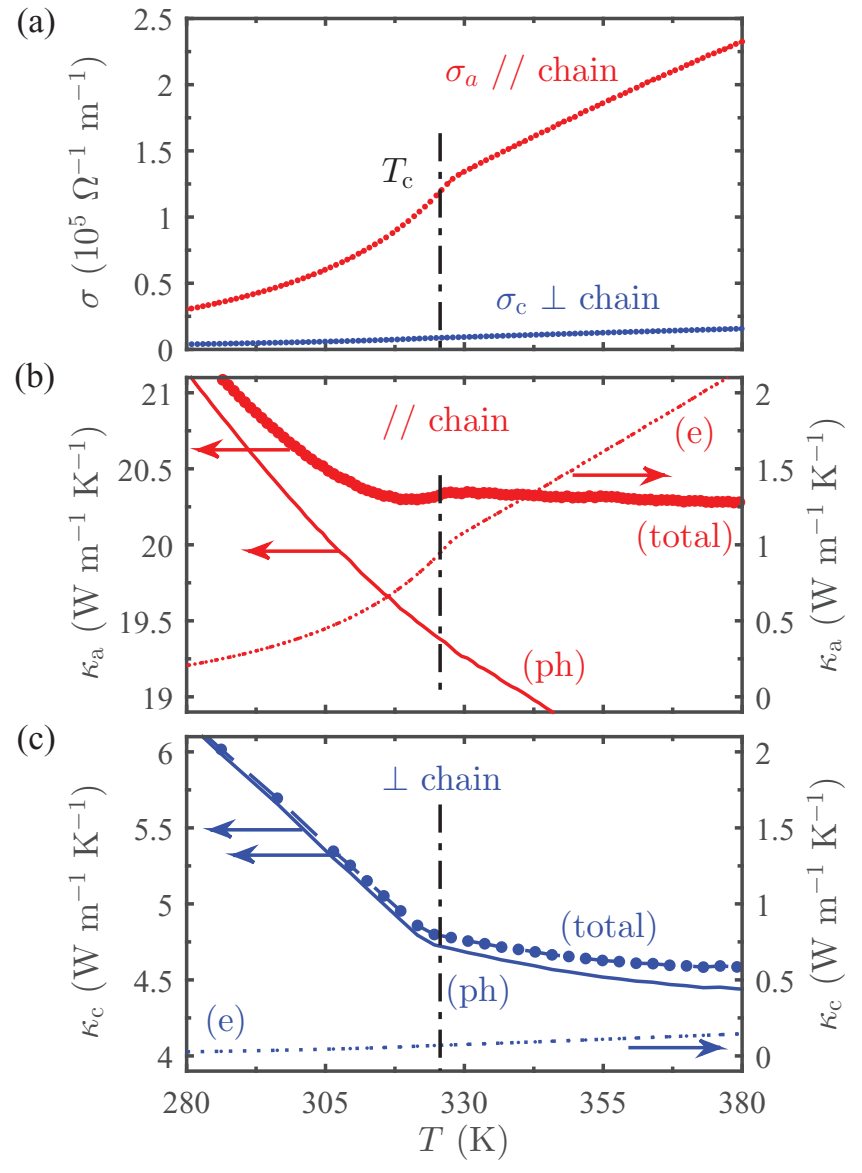

FIG. 2. Separation of electron and phonon contributions to the thermal conductivities $\kappa_{\mathrm{a}}$ with heat current parallel to the chains (\| $a$; red) and $\kappa_{\mathrm{c}}$ with heat current perpendicular to the chains ( $\| c$; blue) in Fig. 1, using the W-F law. (a) The temperature-dependent electrical conductivities, $\sigma_{\mathrm{a}}(T)$ (red) parallel to the chains and $\sigma_{\mathrm{c}}(T)$ (blue) perpendicular to the chains. (b) The total, electron, and phonon thermal conductivities along the $a$ axis, parallel to the chains, are denoted by $\kappa_{\mathrm{a}}$ (circles), $\kappa_{\mathrm{a}}^{(\mathrm{e})}$ (broken), and $\kappa_{\mathrm{a}}^{(\mathrm{ph})}$ (solid), respectively. (c) The total, electron, and phonon thermal conductivities along the $c$ axis, perpendicular to the chains, are denoted $\kappa_{\mathrm{c}}$ (circles), $\kappa_{\mathrm{c}}^{(\mathrm{e})}$ (broken), and $\kappa_{\mathrm{c}}^{(\mathrm{ph})}$ (solid), respectively.

scattering dominates at high temperature [34,35], especially in such a low carrier density system. Then $\kappa^{(\mathrm{ph})}$ can be estimated as the remnant $\kappa-\kappa^{(\mathrm{e})}$. As shown in Fig. 2(a), the electrical conductivity along the chains, $\sigma_{\mathrm{a}}$, is an order of magnitude larger than that perpendicular to the chains, $\sigma_{\mathrm{c}}$, reflecting the quasi-one-dimensional band structure of $\mathrm{Ta}_{2} \mathrm{NiSe}_{5}$. In addition, the anomaly at $T_{\mathrm{c}}$ is much more pronounced in $\sigma_{\mathrm{a}}(T)$ as compared with $\sigma_{\mathrm{c}}(T)$. A much larger electronic contribution to the temperature dependence of $\kappa_{\mathrm{a}}(T)$ than $\kappa_{\mathrm{c}}(T)$ is thus expected.

As shown in Fig. 2(b), the electronic thermal conductivity along the chains $\kappa_{\mathrm{a}}^{(\mathrm{e})}(T)$, estimated from $\sigma_{\mathrm{a}}$, is only $\sim 5 \%$ of the total $\kappa_{\mathrm{a}}(T)$, but fully accounts for the shoulder structure in $\kappa_{\mathrm{a}}(T)$ with the rapid decrease below $T_{\mathrm{c}}$. On the other hand, the phonon thermal conductivity $\kappa_{\mathrm{a}}^{(\mathrm{ph})}(T)$, estimated by subtracting $\kappa_{\mathrm{a}}^{(\mathrm{e})}(T)$ from $\kappa_{\mathrm{a}}(T)$, is surprisingly featureless and shows a monotonic increase through $T_{\mathrm{c}}$, indicating that the anomaly at $T_{\mathrm{c}}$ in $\kappa_{\mathrm{a}}(T)$ is purely electronic in origin. The rapid decrease below $T_{\mathrm{c}}$ therefore reflects the decrease in the number of quasiparticles (electrons and holes), analogous to the prototypical behavior of thermal conductivity in a superconductor [36,37]. We therefore conclude that the quasiparticle transport of a putative EI is captured by thermal transport.

In superconductors, the thermal conductivity sometimes does not show a rapid decrease right below $T_{\mathrm{c}}$, the prototypical behavior, but instead shows an increase $[38,39]$. This can be ascribed to the suppression of scattering of electrons and phonons by electrons due to the opening of an electronic gap below $T_{\mathrm{c}}$. In certain strongly correlated superconductors with anisotropic pairing, such as high- $T_{\mathrm{c}}$ cuprates [38], the enhancement of quasiparticle lifetime due to the suppression of strong electron-electron scattering overcomes the decrease of quasiparticle density and thereby results in an increase of $\kappa$ right below $T_{\mathrm{c}}$. In certain conventional electron-phonon superconductors, the increase of phonon mean-free path due to the suppression of strong scattering of phonons by electrons may also result in the enhancement of phonon thermal conductivity below $T_{\mathrm{c}}$, which brings about the increase of $\kappa$ right below $T_{\mathrm{c}}$ [39]. The observation of a rapid decrease in $\kappa_{\mathrm{a}}(T)$ below $T_{\mathrm{c}}$ in $\mathrm{Ta}_{2} \mathrm{NiSe}_{5}$ thus indicates that the scattering of phonons and electrons with momentum parallel to the chains by electrons should be reasonably weak as compared with other dominant scattering mechanisms, such as phonon-phonon scattering for phonon transport.

As $\sigma_{\mathrm{c}}$ is one order of magnitude smaller and exhibits a much weaker structure at $T_{\mathrm{c}}$ than $\sigma_{\mathrm{a}}$, the electronic thermal conductivity $\kappa_{\mathrm{c}}^{(\mathrm{e})}(T)$ perpendicular to the chain direction is as small as $\sim 1 \%$ of the total $\kappa_{\mathrm{c}}(T)$ in magnitude and, more importantly, does not contribute appreciably to the kinklike anomaly at $T_{\mathrm{c}}$ in the temperature dependence of $\kappa_{\mathrm{c}}(T)$, as seen in Fig. 2(c). In contrast to $\kappa_{\mathrm{a}}(T)$ along the chains, $\kappa_{\mathrm{c}}(T)$ around $T_{\mathrm{c}}$ is dominated by $\kappa_{\mathrm{c}}^{(\mathrm{ph})}(T)$ and is almost purely phononic in origin. The comparison of $\kappa_{\mathrm{c}}^{(\mathrm{ph})}(T)$ and $\kappa_{\mathrm{a}}^{(\mathrm{ph})}(T)$ reveals a substantial anisotropy not only in electron transport but also in phonon transport between the directions parallel and perpendicular to the chains. As clearly seen in Fig. 3, while a $1 / T$-like monotonous decrease with featureless behavior around $T_{\mathrm{c}}$ is observed in $\kappa_{\mathrm{a}}^{(\mathrm{ph})}(T)$ (\| chain), a clear $\mathrm{V}$-shaped suppression from the $1 / T$-like decrease centered at $T_{\mathrm{c}}$ is seen in $\kappa_{\mathrm{c}}^{(\mathrm{ph})}(T)$ ( $\perp$ chain). The suppression is broad in temperature range and appears to be asymmetric above and below $T_{\mathrm{c}}$. While $\kappa_{\mathrm{c}}^{(\mathrm{ph})}(T)$ shows a rapid increase and crossover to $1 / T$-like behavior with decreasing temperatures right below $T_{\mathrm{c}}$, it is almost flat up to at least $400 \mathrm{~K}$ with increasing temperature from $T_{\mathrm{c}}$, implying the persistence of strong suppression of out-of-chain phonon conduction over a wide temperature range above $T_{\mathrm{c}}$.

Phonon thermal conductivity is generally expressed as $\kappa^{(\mathrm{ph})}=\frac{1}{3} C^{(\mathrm{ph})} v^{(\mathrm{ph})} l^{(\mathrm{ph})}$, where $C^{(\mathrm{ph})}, v^{(\mathrm{ph})}$, and $l^{(\mathrm{ph})}$ are the specific heat, the velocity, and the mean-free path of phonons [34], and specifically for $\mathrm{Ta}_{2} \mathrm{NiSe}_{5}$ many acoustic and optical phonon branches are expected to contribute to $\kappa^{(\mathrm{ph})}$ around $T_{\mathrm{c}}[20,24]$. The temperature dependence of the total heat capacity $C$ for $\mathrm{Ta}_{2} \mathrm{NiSe}_{5}$ around $T_{\mathrm{c}}=326 \mathrm{~K}$ is rather small and the small anomaly at $T_{\mathrm{c}}$ is highly likely to be purely electronic in origin [8]. The temperature dependence 


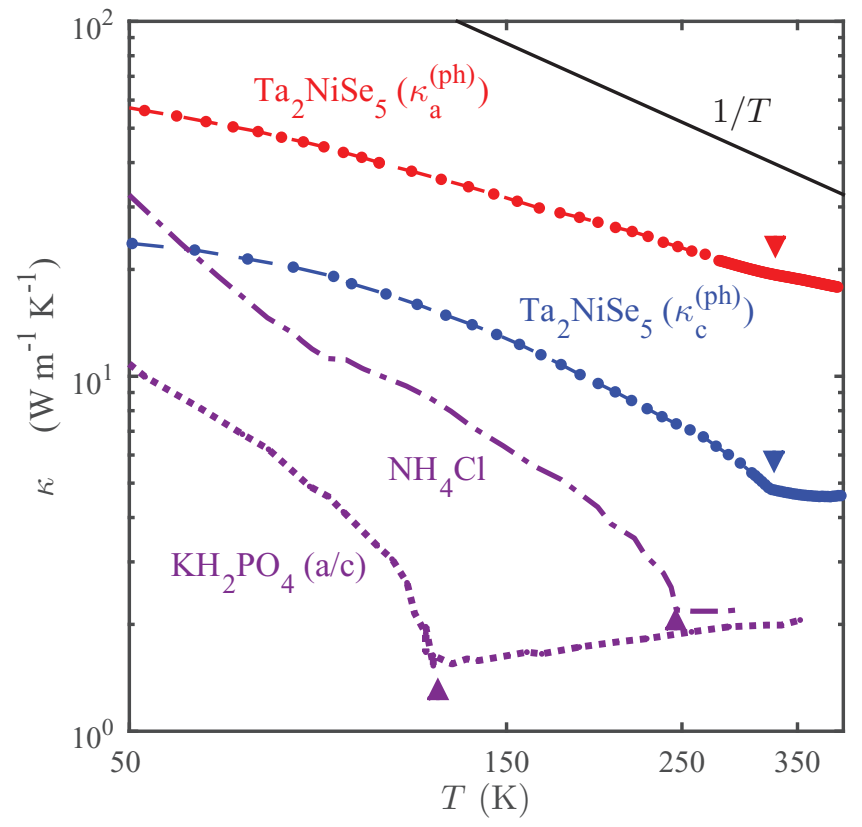

FIG. 3. Comparison of temperature-dependent thermal conductivities $\kappa(T)$ for $\mathrm{Ta}_{2} \mathrm{NiSe}_{5}$ (\| $a$ red; $\| c$ blue) with typical orderdisorder-type ferroelectrics, $\mathrm{NH}_{4} \mathrm{Cl}$ (reproduced from [40]), and $\mathrm{KH}_{2} \mathrm{PO}_{4}$ (reproduced from [41]). The solid black line is a guide line for the $1 / T$ power law.

of $C^{(\mathrm{ph})}$ can thus be ignored in discussing the origin of the V-shaped suppression of $\kappa_{\mathrm{c}}^{(\mathrm{ph})}(T)$. The suppression of $\kappa_{\mathrm{c}}^{(\mathrm{ph})}(T)$ should originate instead from the reduction of $v^{(\mathrm{ph})}$ and/or $l^{(\mathrm{ph})}$ around $T_{\mathrm{c}}$. As it occurs only for phonons with momentum q $\| c$ and phonon scattering should dominate $\kappa_{\mathrm{c}}^{(\mathrm{ph})}(T)$ at high temperatures around $T_{\mathrm{c}}$, it is natural to ascribe its origin to the reported softening of the acoustic shear-mode phonon with $\mathbf{q} \| c$, which corresponds to the monoclinic distortion of lattice frozen at the transition $T_{\mathrm{c}}=326 \mathrm{~K}$ [24]. As pronounced softening occurs only for one specific acoustic phonon mode, the reduction of $v^{(\mathrm{ph})}$ is unlikely to account for the large suppression of $\kappa_{\mathrm{c}}^{(\mathrm{ph})}(T)$ at $T_{\mathrm{c}}$ [Fig. 2(c)]. The primary origin of the suppression of $\kappa_{\mathrm{c}}^{(\mathrm{ph})}(T)$ is highly likely the increased scattering of phonons, namely, the reduction of $l^{(\mathrm{ph})}$ by the soft acoustic shear-mode phonon. The strong suppression above $T_{\mathrm{c}}$ up to at least $400 \mathrm{~K}$ should then imply the robust softening of the shear mode acoustic phonon above $T_{\mathrm{c}}$, which is indeed quite consistent with the reported phonon dispersion relationship of $\mathrm{Ta}_{2} \mathrm{NiSe}_{5}$ measured by inelastic $\mathrm{x}$-ray scattering up to $400 \mathrm{~K}$ [24].
We should point out that the asymmetric V-shaped suppression of $\kappa_{\mathrm{c}}^{(\mathrm{ph})}(T)$, more robust above $T_{\mathrm{c}}$, is analogous to those observed around the order-disorder-type ferroelectric transition in $\mathrm{KH}_{2} \mathrm{PO}_{4}$ and $\mathrm{NH}_{4} \mathrm{Cl}$, as shown in Fig. 3. The order-disorder-type ferroelectrics have extended ferroelectric fluctuations above $T_{\mathrm{c}}$, where well-developed but fluctuating local dipoles give rise to soft local phonons and enhanced phonon scattering over a wide temperature range and hence suppress $\kappa(T)$ strongly over a wide temperature range above $T_{\mathrm{c}}[28,40-42]$. It is tempting to infer that, analogous to the order-disorder-type ferroelectrics, large and local excitonic and/or hybridization gap $(\mathbf{q}=0$ charge density wave) fluctuations may be present above $T_{\mathrm{c}}$ in $\mathrm{Ta}_{2} \mathrm{NiSe}_{5}$. The electronic fluctuations are inherently and strongly coupled to the shear mode phonon, which may give rise to the extended phonon softening above $T_{c}$ and may suppress $\kappa_{\mathrm{c}}^{(\mathrm{ph})}(T)$ over a wide temperature range. Such giant excitonic and/or hybridization gap fluctuations may be inferred from the temperature dependence of the bulk magnetic susceptibility $\chi(T)$ of $\mathrm{Ta}_{2} \mathrm{NiSe}_{5}$ [22], where $\chi(T)$ at temperatures well above $T_{\mathrm{c}}$ shows a pronounced downturn on lower temperatures toward $T_{\mathrm{c}}$, indicative of such fluctuations.

In summary, we have discovered an extremely anisotropic behavior of the thermal conductivity around the putative excitonic transition in the layered chalcogenide $\mathrm{Ta}_{2} \mathrm{NiSe}_{5}$. The distinct temperature-dependent in-plane $\kappa_{\mathrm{a}}(T)$ and $\kappa_{\mathrm{c}}(T)$, parallel and perpendicular to the Ta and Ni chains, respectively, allow us to unveil the behaviors of quasiparticles (electrons and holes) and phonons around the transition temperature $T_{\mathrm{c}}=326 \mathrm{~K}$. The rapid suppression in the number of quasiparticles below $T_{\mathrm{c}}$, analogous to superconductors, is captured in $\kappa_{\mathrm{a}}(T)$ due to the quasi-one-dimensional electronic structure and the $\mathbf{q} \| a$ phonons being insensitive to the transition. The soft shear-mode acoustic phonons with momentum $\mathbf{q} \| c$, which correspond to the monoclinic structural distortion below $T_{\mathfrak{c}}$, manifest themselves only in $\kappa_{\mathfrak{c}}(T)$ perpendicular to the chains as a pronounced suppression of dominant phonon thermal transport. The suppression of $\kappa_{\mathrm{c}}$ extends up to high temperatures well above $T_{\mathrm{c}}$, indicating the persistence of phonon softening over a wide temperature range. This may suggest the presence of giant excitonic and/or hybridization gap fluctuations above $T_{\mathrm{c}}$ and the strong electron-lattice coupling for the monoclinic distortion in $\mathrm{Ta}_{2} \mathrm{NiSe}_{5}$.

This work is supported in part by the Alexander von Humboldt Foundation. We thank L. Dorner-Finkbeiner and C. Bush for experimental support in crystal growth and characterization, A. Bangura for suggesting the "heat-pipe" technique, and D. Huang for useful discussions.
[1] D. Jérome, T. M. Rice, and W. Kohn, Phys. Rev. 158, 462 (1967).

[2] P. Wachter, B. Bucher, and J. Malar, Phys. Rev. B 69, 094502 (2004).

[3] A. Kogar, M. S. Rak, S. Vig, A. A. Husain, F. Flicker, Y. I. Joe, L. Venema, G. J. MacDougall, T. C. Chiang, and E. Fradkin, Science 358, 1314 (2017).

[4] P. Wachter, Adv. Mater. Phys. Chem. 8, 120 (2018).
[5] F. J. Di Salvo, D. E. Moncton, and J. V. Waszczak, Phys. Rev. B 14, 4321 (1976).

[6] Y. Wakisaka, T. Sudayama, K. Takubo, T. Mizokawa, M. Arita, H. Namatame, M. Taniguchi, N. Katayama, M. Nohara, and H. Takagi, Phys. Rev. Lett. 103, 026402 (2009).

[7] S. Kim, Y. Kim, C. Kang, E. An, H. K. Kim, M. J. Eom, M. Lee, C. Park, T. Kim, H. Choi, B. Min, and J. Kim, ACS Nano 10, 8888 (2016). 
[8] Y. F. Lu, H. Kono, T. I. Larkin, A. W. Rost, T. Takayama, A. V. Boris, B. Keimer, and H. Takagi, Nat. Commun. 8, 14408 (2017).

[9] T. I. Larkin, A. N. Yaresko, D. Pröpper, K. A. Kikoin, Y. F. Lu, T. Takayama, Y.-L. Mathis, A. W. Rost, H. Takagi, B. Keimer, and A. V. Boris, Phys. Rev. B 95, 195144 (2017).

[10] S. Mor, M. Herzog, D. Golež, P. Werner, M. Eckstein, N. Katayama, M. Nohara, H. Takagi, T. Mizokawa, C. Monney, and J. Stähler, Phys. Rev. Lett. 119, 086401 (2017).

[11] D. Werdehausen, T. Takayama, M. Höppner, G. Albrecht, A. W. Rost, Y. Lu, D. Manske, H. Takagi, and S. Kaiser, Sci. Adv. 4, eaap8652 (2018).

[12] Y. Seo, M. Eom, J. Kim, C. Kang, B. I. Min, and J. Hwang, Sci. Rep. 8, 11961 (2018).

[13] K. Okazaki, Y. Ogawa, T. Suzuki, T. Yamamoto, T. Someya, S. Michimae, M. Watanabe, Y. Lu, M. Nohara, H. Takagi, N. Katayama, H. Sawa, M. Fujisawa, T. Kanai, N. Ishii, J. Itatani, T. Mizokawa, and S. Shin, Nat. Commun. 9, 4322 (2018).

[14] K. Fukutani, R. Stania, J. Jung, E. F. Schwier, K. Shimada, C. I. Kwon, J. S. Kim, and H. W. Yeom, Phys. Rev. Lett. 123, 206401 (2019).

[15] L. Chen, T. T. Han, C. Cai, Z. G. Wang, Y. D. Wang, Z. M. Xin, and Y. Zhang, Phys. Rev. B 102, 161116(R) (2020).

[16] P. A. Volkov, M. Ye, H. Lohani, I. Feldman, A. Kanigel, K. Haule, and G. Blumberg, npj Quantum Mat. 6, 52 (2021).

[17] K. Kim, H. Kim, J. Kim, C. Kwon, J. S. Kim, and B. J. Kim, Nat. Commun. 12, 1969 (2021).

[18] Q. He, X. Que, L. Zhou, M. Isobe, D. Huang, and H. Takagi, arXiv:2012.08395 [cond-mat.str-el].

[19] M. D. Watson, I. Marković, E. A. Morales, P. Le Fèvre, M. Merz, A. A. Haghighirad, and P. D. C. King, Phys. Rev. Res. 2, 013236 (2020).

[20] A. Subedi, Phys. Rev. Mater. 4, 083601 (2020).

[21] E. Baldini, A. Zong, D. Choi, C. Lee, M. H. Michael, L. Windgaetter, I. I. Mazin, S. Latini, D. Azoury, B. Lv, A. Kogar, Y. Wang, Y. Lu, T. Takayama, H. Takagi, A. J. Millis, A. Rubio, E. Demler, and N. Gedik, arXiv:2007.02909 [cond-mat.str-el].

[22] F. J. Di Salvo, C. H. Chen, R. M. Fleming, J. V. Waszczak, R. G. Dunn, S. A. Sunshine, and J. A. Ibers, J. Less-Common Met. 116, 51 (1986).
[23] A. Nakano, K. Sugawara, S. Tamura, N. Katayama, K. Matsubayashi, T. Okada, Y. Uwatoko, K. Munakata, A. Nakao, and H. Sagayama, IUCrJ 5, 158 (2018).

[24] A. Nakano, T. Hasegawa, S. Tamura, N. Katayama, S. Tsutsui, and H. Sawa, Phys. Rev. B 98, 045139 (2018).

[25] G. Mazza, M. Rösner, L. Windgätter, S. Latini, H. Hübener, A. J. Millis, A. Rubio, and A. Georges, Phys. Rev. Lett. 124, 197601 (2020).

[26] M. Tinkham, Introduction to Superconductivity, 2nd ed. (McGraw-Hill, Inc., New York, 1996).

[27] S.-M. Lee, S. Lim, S.-I. Kwun, and Y. H. Jeong, Solid State Commun. 88, 361 (1993).

[28] B. A. Strukov and A. A. Belov, Phase Transit. 51, 175 (1994).

[29] J. Zittartz, Phys. Rev. 165, 612 (1968).

[30] See Supplemental Material at http://link.aps.org/supplemental/ 10.1103/PhysRevB.104.L121201 for further information regarding crystal preparation, electrical resistivity, temperature derivatives of thermal conductivities, phonon heat capacity, and phonon thermal diffusivity.

[31] P. B. Allen, X. Du, L. Mihaly, and L. Forro, Phys. Rev. B 49, 9073 (1994).

[32] N. Wakeham, A. F. Bangura, X. Xu, J.-F. Mercure, M. Greenblatt, and N. E. Hussey, Nat. Commun. 2, 1 (2011).

[33] K. Momma and F. Izumi, J. Appl. Crystallogr. 44, 1272 (2011).

[34] R. Berman, Thermal Conduction in Solids (Clarendon Press, Oxford, 1976).

[35] T. M. Tritt (Ed.), Thermal Conductivity: Theory, Properties, and Applications (Springer Science \& Business Media, Boston, MA, 2005).

[36] C. B. Satterthwaite, Phys. Rev. 125, 873 (1962).

[37] V. Ambegaokar and J. Woo, Phys. Rev. 139, A1818 (1965).

[38] R. C. Yu, M. B. Salamon, J. P. Lu, and W. C. Lee, Phys. Rev. Lett. 69, 1431 (1992).

[39] J. R. Carlson and C. B. Satterthwaite, Phys. Rev. Lett. 24, 461 (1970).

[40] W. Bausch and W. Waidelich, Phys. Lett. A 30, 190 (1969).

[41] Y. Suemune, J. Phys. Soc. Jpn. 22, 735 (1967).

[42] M. E. Lines and A. M. Glass, Principles and Applications of Ferroelectrics and Related Materials (Oxford University Press, New York, 2001). 